\title{
Test Result Utilization in the Development Process of High Voltage SF6 Circuit-Breaker
}

\author{
Zsolt Mitrik, Péter Kádár \\ Óbuda University, Kálmán Kandó Faculty of Electrical Engineering, \\ Power System Department, Alternative Eneregy Sources Laboratory \\ Bécsi út 94-96, H-1034 Budapest, Hungary \\ mitrik.zsolt@kvk.uni-obuda.hu; kadar.peter@kvk.uni-obuda.hu
}

\begin{abstract}
During the development tests of high voltagecircuit-breakers (HVCB), the manufacturers obtain a large amount of information regarding the behavior and parameters of the equipment. Such valuable conclusions can be deduced from processing, analyzing and evaluating them by scientific methods that are appropriate for developing a new decision-supporting model. The Author describes the methodology of the development process of such part of the new decision-supporting model (Circuit Breaker Design algorithm $\mathrm{CBDa}$ ) which systematizes and evaluates the information from tests as results and illustrates the possible ways of usage.
\end{abstract}

Keywords: HVCB; $3^{\text {rd }}$ generation SF6 circuit-breaker; CBDa model

\section{Introduction}

High-voltage circuit-breakers are an integral part of the electricity system. Their task is to close and open the circuits in normal operational and outside of normal operational circumstances. The circuit-breakers are the only short circuitprotection devices (SCPD) in the high voltage networks. Accordingly, reliable operation is critical in terms of reliability of the entire system. It is necessary to extinguish the arc between the contacts that appears during the opening process, within the shortest time, even several times in succession at the breaking of the short-circuit currents. The arc chambers built into the circuit-breaker perform this task [1]. The Author analyses those high voltage circuit-breakers that use SF6 gas as arc extinguisher medium, which is the same as the insulator medium. The Author investigates the third generation, auto-puffer high voltage circuit-breakers, as those are the most common and uses the most modern technology. The IEC 62271-100:2008/AMD2:2017/COR1:2018 [2] refers to high voltage circuitbreakers and describes what types of breaking tests certify that the tested circuitbreaker is able to perform its tasks under any network conditions and would be able to break the circuit safely. During the design of the circuit-breakers, the 
designers follow two main directions. Firstly, they perform a computer simulation in which they analyze the variation of the generated gas flow (speed, density, pressure change) and the electrical field strengthen the developed geometric circumstances [3]. On the other hand, based on the results of the simulations, test equipment is manufactured on which the designers carry out the tests in order to verify the correctness of the calculations. The Author created and introduced to the science a new Circuit Breaker Design algorithm (CBDa) model [4] as a result of the Author's scientific work which is suitable for modeling the SF6 high voltage circuit-breaker development process. Figure 1 shows the CBDa model's main steps:

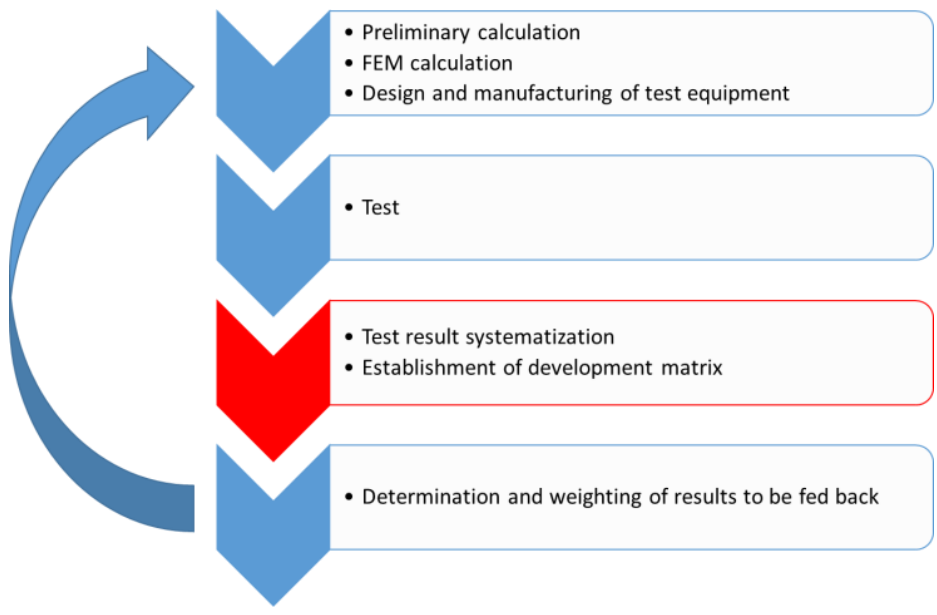

Figure 1

Simplified flow chart of CBDa model

This article describes the third step of Figure 1 in detail. In the following chapters, the Author presents the evaluation of results and information from the completed tests and the utilization of them. The fourth step of the model (Figure 1) is the determination and weighting of results to be fed back [5]. The author will describe the feedback method in then extarticle. It will also include the methodology of weighting and how all these increase the accuracy of preliminary calculations and simulations.

\section{Theory and Process of Measurement}

The measurement starts with the determination of the scope of the measurement. The first step is to determine what one would find out with the measurement. The second step of the measurement process is the collection of all prior information and knowledge. It is investigated what other researchers used what variables and 
which methods and what results they reached. After all these, one plans the measurement process: what is measured, which equipment is used for the measure, what protocol will apply for the tests. At the next step, the measurement equipment is established and the measurement instrument is checked [6]. The actual measurement/test is processed after this and if needed, correction can be set up in the measurement process. The final step of the measurement process is the evaluation of the test results. [7]

\subsection{Application of the Measurement Theory}

The measurement theory [8] is a scientific tool used during the measurement process: measurement planning, data collection, measurement and evaluation of results. It can be applied and performed step-by-step: (Fig. 2).
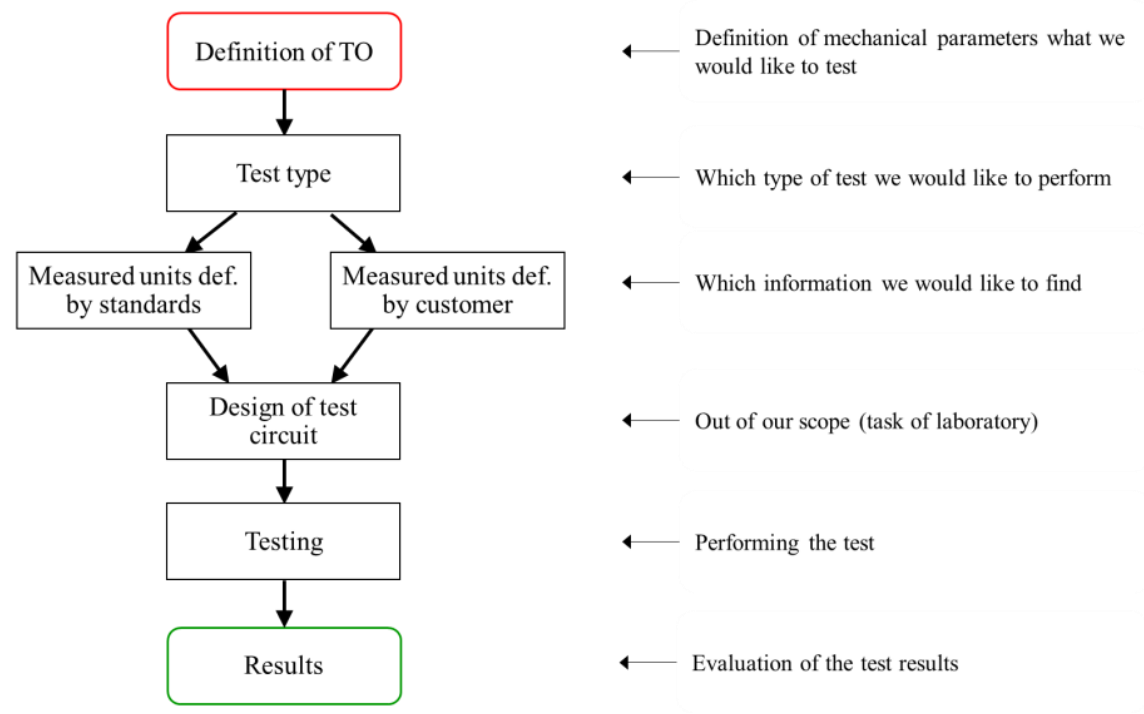

Figure 2

Circuit-breaker test flow chart

Figure 2 shows the test process. The first step is the determination of the geometry to be tested, i.e. the assembly of the test circuit-breaker (Test object - TO). In the next step it is determined in accordance with the CBDa model [4] and the standards (2.2) which test would be performed. Then, the researcher defines the measurable physical quantities. The standards prescribe part of these (typically the electrical parameters of the test circuit). However, such additional parameters can be determined that can be used at a later stage also for feedback. The test laboratory can design their own test circuit once the measurement circuit and supplementary parameters are determined. The tests can be performed after these steps. The Author describes the evaluation of the results in the last chapter (4). 


\subsection{Standard Environment of Synthetic Tests}

The IEC 62271-100:2008/AMD2:2017/COR1:2018 [2] applies to the high voltage circuit-breakers. The standard prescribes the carry-out of all such test that needs for the certification that the high voltage circuit-breaker can be built into the system, and also for the proof of the proper operation in the system and for the proof of the lifetime. In this article, the author deals only with the breaking tests and the evaluation of such tests. The fulfillment of the following tests prove that the circuit-breaker operates properly under any circuit circumstances:

1. Basic short-circuit tests (T100s)

2. Basic short-circuit tests (T100a)

3. Basic short-circuit tests (T60)

4. Basic short-circuit tests (T30)

5. Basic short-circuit tests (T10)

6. Short-line fault test (L90)

7. Short-line fault test (L75)

8. Out-of-phase making and breaking test (OP2)

9. Capacitive current switching test (BC2) Making / Breaking

10. Capacitive current switching test (BC1)

11. Double-earth fault test (DEF)

One part of the above tests are opening tests $(\mathrm{O})$, other parts are closing and opening tests $(\mathrm{CO})$ and there are tests to simulate the opening - closing- opening operations $(\mathrm{O}-0,3 \mathrm{~s}-\mathrm{CO})$ with that the fast reclose automation is simulated.

Certain tests must be performed with different arcing times. The minimum arcing time in this context is the shortest time under which the circuit-breaker in the set circuit conditions can interrupt the circuit. Some tests are performed with maximum arcing time, others with medium arcing time and there are combinations of these. The standards prescribe that the minimum arcing time must be found first and all other test times must be calculated from this value.

\subsection{The Process of Measurement}

Due to the high energy need of the above-described tests, it is not possible to perform the test on the electric network. So-called synthetic circuits are used for these tests. Figure 3 shows a sample synthetic circuit. [9] 


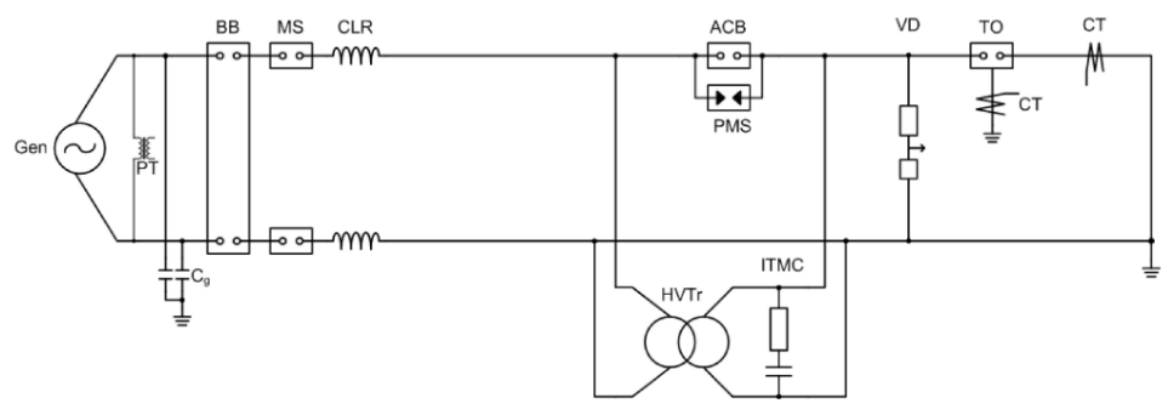

Figure 3

Test circuit for basic short-circuit tests (T100s(a)) [10]

The Figure 3 shows the simplified schematic connection drawing of a synthetic circuit that is suitable for T100s (a) test. Other, similar circuits are assigned to each test types. The characteristics of the network - the test laboratory wants to simulate - can be reached by the fine-tuning of the built-in inductive and capacitive elements. [11]

On Figure 3 there are the following marks: Gen - generator, PT - potential transformer, Cg - TRV tuning capacitor, BB - back-up circuit-breaker, MS making switch, CLR - current limiting reactor, ACB - auxiliary circuit-breaker, PMS - Plasma making switch, VD - voltage divider, TO - test object, CT current transformer, HVTr - high voltage transformer, ITMC - Initial transient making current circuit.

The standards prescribe that the minimum arcing time must be found first and all other test times must be calculated from this value. However, this means that tests must be carried out on the circuit-breaker in a way that the arcing time is decreased by milliseconds until the circuit-breaker cannot interrupt the circuit. Accordingly, a circuit-breaker carries out more breaks even before the real tests. This fact is important as certain assembled parts are damaged or worn with each breaking. This worn may affect the arcing contacts and the material (Teflon) of the gas deflector components (e.g. main nozzles) around the arcing place which evaporates due to the extreme heat. As a consequence of the evaporation of this material, the geometry of the nozzles changes and this decreases the efficiency of the arc extinguish. Figure 4 shows the simplified assembly of the arcing chamber of an SF6 circuit-breaker.

Figure 4 shows an arcing chamber of the circuit-breaker in which 1 is the gas deflector, 2 and 6 are arcing contacts, 3 - arc formation place, 4, 5 - main contacts. 


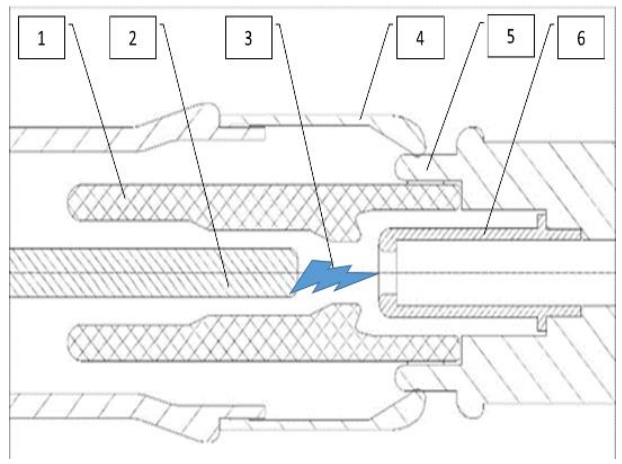

Figure4

Simplified assembly of circuit-breaker arcing chamber (own figure)

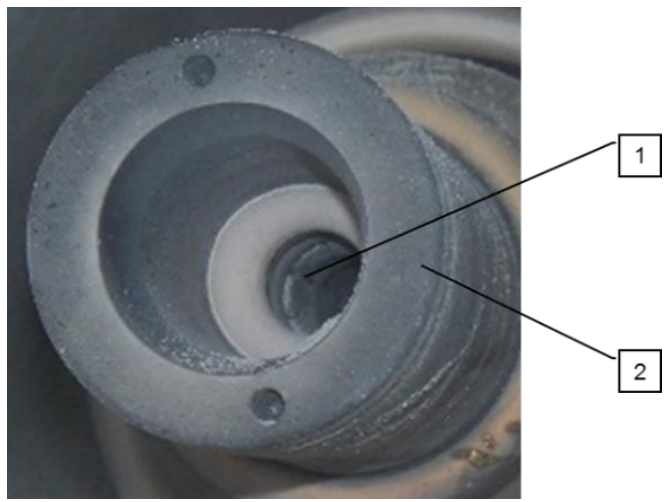

Figure 5

Part of used arcing chamber (own photo)

Figure 5is a picture about a part of a used arcing chamber. It can be observed that the arcing sleeve (1) and the main nozzle (2) melted and evaporated due to the heat.

During the developmental process, several geometries is tested. These typically relate to the volume of the thermal and compression chambers, the establishment of that geometry that determines the gas flow channel. Generally, the testing process starts with tests that load the circuit-breaker the most. These are the $100 \%$ breaking and the L90 test duties. At the same, time it is necessary to consider that due to the operational principle of third-generation (auto-puffer) SF6 circuitbreakers, the range of medium short-circuit currents, test duty (T60), represents a significant strain [12]. If the first test on the geometry established by the computer simulation is successful then the next test is carried out on the same geometry. During the test a large amount of information is obtained on the characteristics of the circuit-breaker. The evaluation and feedback of this information into the development process may increase the accuracy and effectiveness of the development. 


\section{The Measurement and the Organization of Measurement Results}

The large amount of data generated during the development process classified into three groups: mechanical parameters (3.1), test parameters (3.2) and the ordering of measurement results (3.3).The aggregated matrix, as the result of the data in the three groups, is presented in the chapter 3.4. [13]

\subsection{Ordering of Mechanical Parameters}

Several parts are assembled in the arcing chambers of third-generation SF6 circuit-breakers. The Author - based on prior experiences - determined sixteen different mechanical parameters (examined geometric shape) which have major impact on the arc extinguish process. These mechanical parameters are marked as $\mathrm{M}_{1}-\mathrm{M}_{16}$.

During the tests the parameters are modified continuously and are summarized in the matrix (1):

$A_{T M}=\left(\begin{array}{cccc}M_{1,1} & M_{1,2} & \ldots & M_{1,16} \\ M_{2,1} & M_{2,2} & \ldots & M_{2,16} \\ \ldots & \ldots & \ldots & \ldots \\ M_{n, 1} & M_{n, 2} & \ldots & M_{n, 16}\end{array}\right)$

where the matrix columns indicate a mechanical parameter and each line corresponds to a test configuration, shape combination.

\begin{tabular}{|c|c|c|c|c|c|c|c|c|c|c|c|c|c|c|c|c|c|}
\hline \multicolumn{2}{|c|}{$\begin{array}{c}\text { Mechanical } \\
\text { parameter }\end{array}$} & M1 & M2 & M3 & M4 & M5 & M6 & M7 & M8 & M9 & M10 & M11 & M12 & M13 & M14 & M15 & M16 \\
\hline \multirow{7}{*}{ 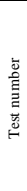 } & 37 & $\ldots$ & $\ldots$ & $\ldots$ & $\ldots$ & $\ldots$ & --- & -.. & $\ldots$ &.-- & $\ldots$ & $\ldots$ & -.. & -.. & $\ldots$ & --- & $\ldots$ \\
\hline & 38 & 2*) & 175 & 24 & 33 & $\ldots$ & $12 / 19$ & $42 / 67$ & 20 & R11 & 3 & normal & 125 & NO & 320 & 500 & 37 \\
\hline & 39 & $2 *)$ & 175 & 24 & 33 & $\ldots$ & $3 / 19$ & $36 / 67$ & 14 & R11 & 3 & normal & 125 & $\mid 866530-\mathrm{I}$ & 320 & 500 & 37 \\
\hline & 40 & 2*) & -.- & $\ldots$ & 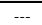 & $-\ldots$ & $-\ldots$ & 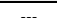 & $\ldots$ & --- & -.- & -.- & -.- & $\ldots$ & $\ldots$ & -.- & $\ldots$ \\
\hline & 41 & 5 & 187 & 24 & 33 & --- & $12 / 19$ & $36 / 79$ & 14 & R11 & 1 & normal & 145 & $866530-\mathrm{I}$ & 320 & 500 & 37 \\
\hline & 42 & --- & $\ldots$ & -- & -.- & $\ldots$ & -.- & -.. & $\ldots$ & $\ldots$ & $-\cdots$ & $\ldots$ & $\ldots$ & -.- & $\ldots$ & -.- & --- \\
\hline & 43 & 4 & 187 & 24 & 34,4 & $\ldots$ & $3 / 19$ & $36 / 79$ & 14 & R11 & 1 & normal & 145 & $866530-\mathrm{I}$ & 320 & 550 & 37 \\
\hline
\end{tabular}

Figure 6

Mechanical data in table format

Lines are taken on till the given test type becomes successful.

\subsection{Ordering of Test Parameters}

According to the above, the test parameters are also included in a matrix (2). $E_{1}$ indicates the test type (Chapter 2 standard environment), $\mathrm{E}_{2}-\mathrm{E}_{9}$ are the measured electric and mechanical values. Even though the standards determine the value of the electric parameters (e.g. transient recovery voltage (TRV) value, rate of decrease of the specified short-circuit current (di/dt), etc.) these can differ from the nominal value due to the standard deviation of the synthetic circuit. Therefore, the 
author considers these values as the measured value. The matrix (2) also contains mechanical values that are typical for that test (e.g. set gas pressure, operational speed and main time of circuit-breaker, etc.):

$A_{T E}=\left(\begin{array}{cccc}E_{1,1} & E_{1,2} & \ldots & E_{1,8} \\ E_{2,1} & E_{2,2} & \ldots & E_{2,8} \\ \ldots & \ldots & \ldots & \ldots \\ E_{n, 1} & E_{n, 2} & \ldots & E_{n, 8}\end{array}\right)$

where the matrix columns indicate a test parameter. Similarly to the mechanical parameters, the lines are taken on till the test type becomes successful.

\begin{tabular}{|c|c|c|c|c|c|c|c|c|c|c|c|}
\hline \multicolumn{2}{|c|}{$\begin{array}{c}\text { Electrical } \\
\text { parameter }\end{array}$} & \multicolumn{2}{|c|}{ E1 } & E2 & E3 & E4 & E5 & E6 & E7 & E8 & E9 \\
\hline \multirow{4}{*}{} & 37 & L90 & AQF0507 & 5,98 & 6 & 22,07 & 124 & 35,6 & 15,93 & --- & 169 \\
\cline { 2 - 13 } & 38 & L90 & AQF0606 & 6 & 6 & 17,4 & 125 & 35,74 & 16,34 & --- & \\
\cline { 2 - 13 } & 39 & L90 & AQF1104 & 6 & 6 & 21,44 & 125 & 35,81 & 16,4 & --- & 170 \\
\cline { 2 - 12 } & 40 & L90 & AQF1106 & 6 & 6 & 24,9 & 125 & 35,7 & 16,35 & --- & \\
\cline { 2 - 11 } & 41 & L90 & AQF2809 & 5,79 & 6 & 14,64 & 123 & 36,3 & 15,8 & --- & 161 \\
\cline { 2 - 11 } & 42 & L90 & AQF2819 & 5,8 & 6 & 21,71 & 125 & 36,06 & 16,39 & --- & \\
\cline { 2 - 11 } & 43 & L90 & AQG0515 & 6 & 6 & 21,7 & 124 & 35,7 & 16,19 & --- & 165 \\
\hline
\end{tabular}

Figure 7

Test parameters in table format

Figure 7 shows an example of the electric parameters of L90 tests. Similar tables are generated to collect the results of each test types that are specified in point 2.2.

\subsection{Ordering of Measurement Results}

$\mathrm{R}_{1}-\mathrm{R}_{11}$ indicates the measurement results. $\mathrm{R}_{1}$ may have two different values, 1 if the test was successful and 0 - if the test failed. $R_{2}-R_{11}$ indicates such kinds of parameters as, e.g. first and second peak value of the pressure in the thermal chamber. This data is also integrated into a matrix (3):

$A_{T R}=\left(\begin{array}{cccc}R_{1,1} & R_{1,2} & \ldots & R_{1,11} \\ R_{2,1} & R_{2,2} & \ldots & R_{2,11} \\ \ldots & \ldots & \ldots & \ldots \\ R_{n, 1}=1 & R_{n, 2} & \ldots & R_{n, 11}\end{array}\right)$

As before, each line in the matrix includes the results of a new test.

\begin{tabular}{|c|c|c|c|c|c|c|c|c|c|c|c|c|}
\hline \multicolumn{2}{|c|}{$\begin{array}{l}\text { Mesurement } \\
\text { results }\end{array}$} & $\mathrm{R} 1$ & $\mathrm{R} 2$ & R3 & R4 & R5 & R6 & R7 & $\mathrm{R} 8$ & R9 & R10 & R11 \\
\hline \multirow{7}{*}{ 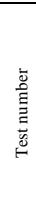 } & 37 & OK/NO & 121 & 3 & $\mathrm{x}$ & X*) & --- & --- & --- & --- & --- & *) $82,5 \mathrm{kV}$, after $6 \mathrm{~ms}$ \\
\hline & 38 & NO & 113 & --- & --- & -.- & 9,42 & --- & 13,03 & 10,7 & --- & --- \\
\hline & 39 & OK & $\ldots$ & $-\ldots$ & --- & --- & 34,2 & $-\ldots$ & 45,7 & 33 & $-\ldots$ & --- \\
\hline & 40 & $\left.\mathrm{NO}^{*}\right)$ & --- & $-\cdots$ & --- & --- & 30 & $\cdots$ & 40,4 & 28,3 & $--\cdot$ & *) membrane breach \\
\hline & 41 & $\mathrm{OK}$ & $-\ldots$ & --- & $\ldots$ & --- & 27,71 & 19,9 & --- & $-\ldots$ & 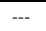 & --- \\
\hline & 42 & NO & $-\cdots$ & $-\ldots$ & --- & $-\ldots$ & 24,26 & 18,5 & 24,16 & 16,36 & $-\ldots$ & $-\ldots$ \\
\hline & 43 & OK/NO & 105 & 16,3 & --- & $\ldots$ & 43,17 & 35,9 & 39,27 & 30,3 & $\ldots$ & $-\ldots$ \\
\hline
\end{tabular}

Figure 8

Measurement results in table format 
Figure 7 and Figure 8 show details of the test parameters and the test results tables. The missing values are such values that are typically non-test data, or data not registered due to measurement circuit failure.

\subsection{The Aggregated Matrix}

\section{Description of the development measurement series}

1. $\mathrm{M}_{1}-\mathrm{M}_{16}$ defines the mechanical characteristics of the equipment. The researcher runs a standard described test type, e.g. L90. This is indicated as T1. It is almost certain that the first test will fail, therefore, $R_{1}$ will be 0 .

2. $\mathbf{M}_{1}-\mathrm{M}_{16}$ values are modified until the test becomes successful. At this time $\mathrm{R}_{1}$ will be 1 .

3. Once $R_{1}=1$, the measurements of next test type $T_{2}$ start. It is also almost certain that the first test is unsuccessful. $\mathbf{M}_{1}-\mathrm{M}_{16}$ values are again modified until $\mathrm{R}_{1}$ becomes 1 .

4. Then the researcher returns to $T_{1}$. Based on the prior measurements, it is clear that what kind of modification of which element of $\mathrm{M}_{1}-\mathrm{M}_{16}$ what effect it has on the result. $M_{1}-M_{16}$ is modified in such a way that $R_{1}=1$, in both $T_{1}$ and $\mathrm{T}_{2}$. This can be reached from several tests.

5. If it is reached that for the same $M_{1}-M_{16}, R_{1}=1$ both at $T_{1}$ and $T_{2}$, then $T_{3}$ can start.

6. This process is continued for all $T_{x}$ until $R_{1}=1$ using the same $M_{1}-M_{16}$ parameters.

7. $R_{2} \ldots R_{11}$ results are used for the determination of the next $M_{1}-M_{16}$ independently from the fact of whether $R_{1}$ value is 0 or 1 .

Aggregating the matrixes of the mechanical parameters, of the test parameter and the matrix of the results, a new matrix is received that is the aggregated matrix of one test type:

$A_{T 1}=\left(\begin{array}{ccccccccccc}M_{1,1} & M_{1,2} & \ldots & M_{1,16} & E_{1,1} & \ldots & E_{1,8} & R_{1,1} & R_{1,2} & \ldots & R_{1,11} \\ M_{2,1} & M_{2,2} & \ldots & M_{2,16} & E_{2,1} & \ldots & E_{2,8} & R_{2,1} & R_{2,2} & \ldots & R_{2,11} \\ \ldots & \ldots & \ldots & \ldots & \ldots & \ldots & \ldots & \ldots & \ldots & \ldots & \ldots \\ M_{n, 1} & M_{n, 2} & \ldots & M_{n, 16} & E_{n, 1} & \ldots & E_{n, 8} & R_{n, 1}=1 & R_{n, 2} & \ldots & R_{n, 11}\end{array}\right)$

Figure 9 shows the application of $\mathrm{A}_{\mathrm{TM}}, \mathrm{A}_{\mathrm{TE}}, \mathrm{A}_{\mathrm{TR}}$ and the aggregated matrix in the previously published CBDa model. The determination of the geometry in the $\mathrm{CBDa}$ model corresponds to a new line of $\mathrm{A}_{\mathrm{TM}}$ matrix. At the same way, the new measured parameters and the measurement results in the tests would increase $A_{\mathrm{TE}}$, $\mathrm{A}_{\mathrm{TR}}$ matrixes. The aggregated matrix represents the amount of information to feedback. 


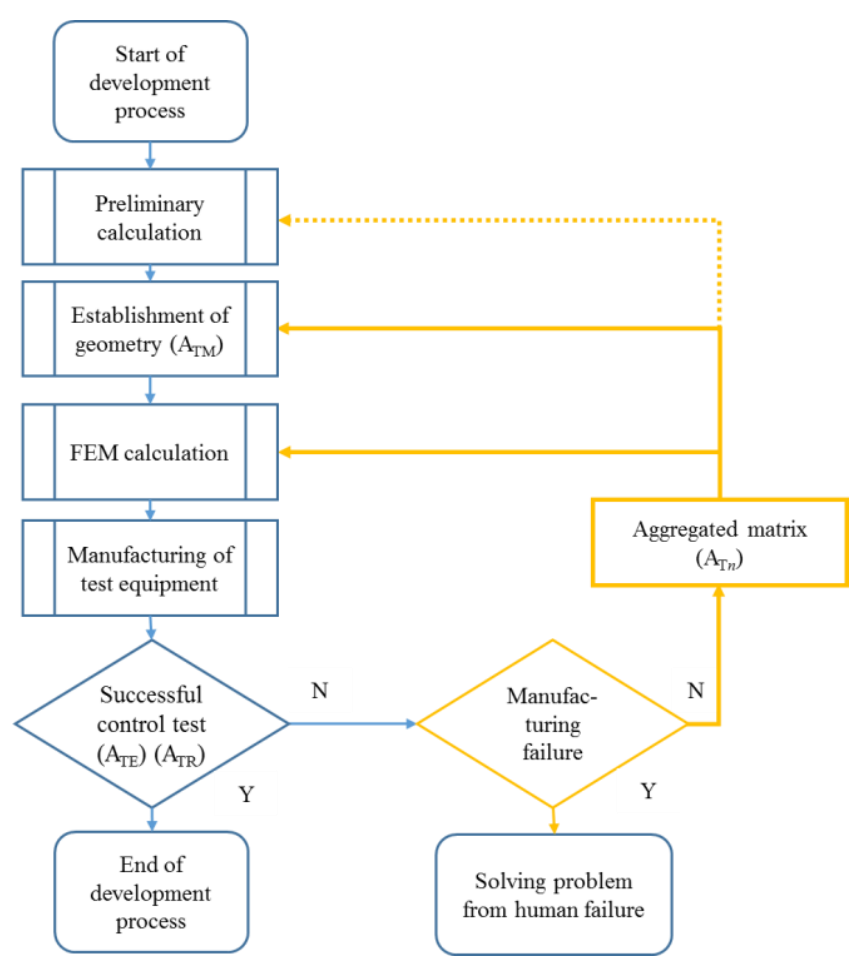

Figure 9

Development process with feedbacks [4]

Figure 9 shows the development process and the integration of the aggregated matrix in the feedback processes. The Authors described the development process in detail in their previously published article. [4]

\section{Evaluation of Feedback Results}

The author, former leader of R\&D team, in Switchgear Department, at Ganz Transelektro Electric Co. took part in several tests performed at VEIKI-VNL Electric Large Laboratories Ltd. Figure 10 and Figure 11 show two test results carried out in this laboratory. The oscillograms contain a completed test result, which is the test of the same circuit-breaker on the same test type, but with different arcing time: 


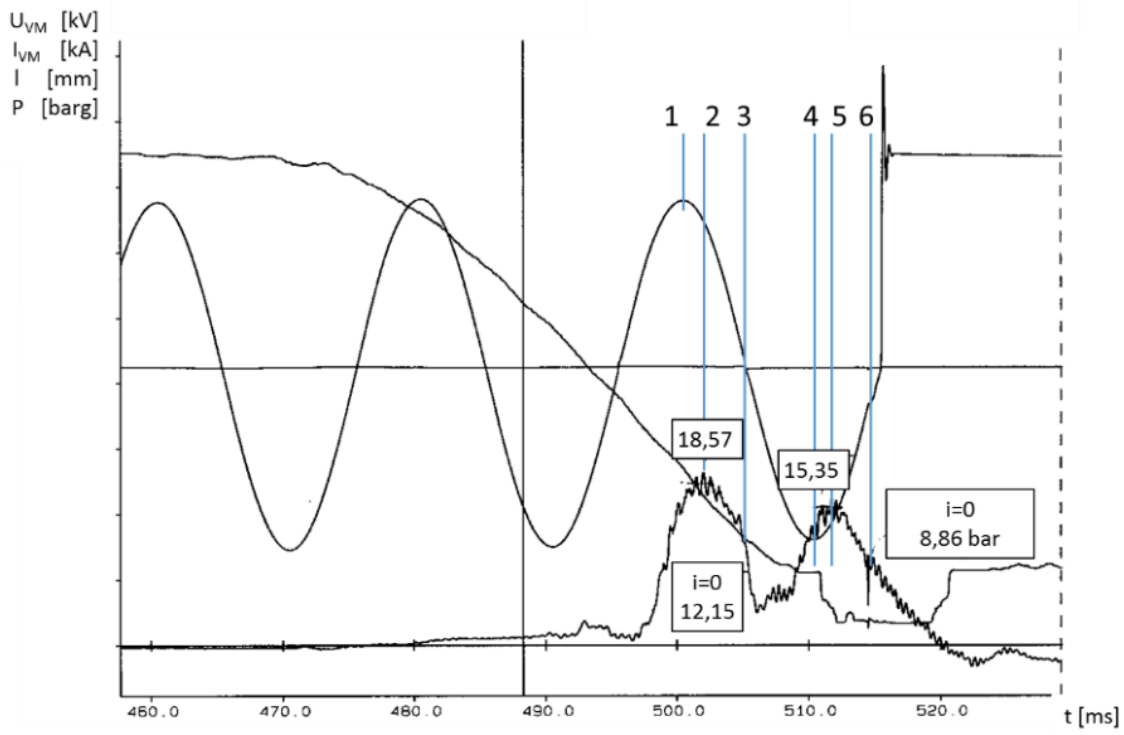

Figure 10

Breaking process with T100s maximum arcing time

Figure 10 shows the current flowing through the poles of the circuit breaker, the voltage between the poles after the current interruption process, the time-travel diagram and the gas pressure evolution in the thermal chamber.

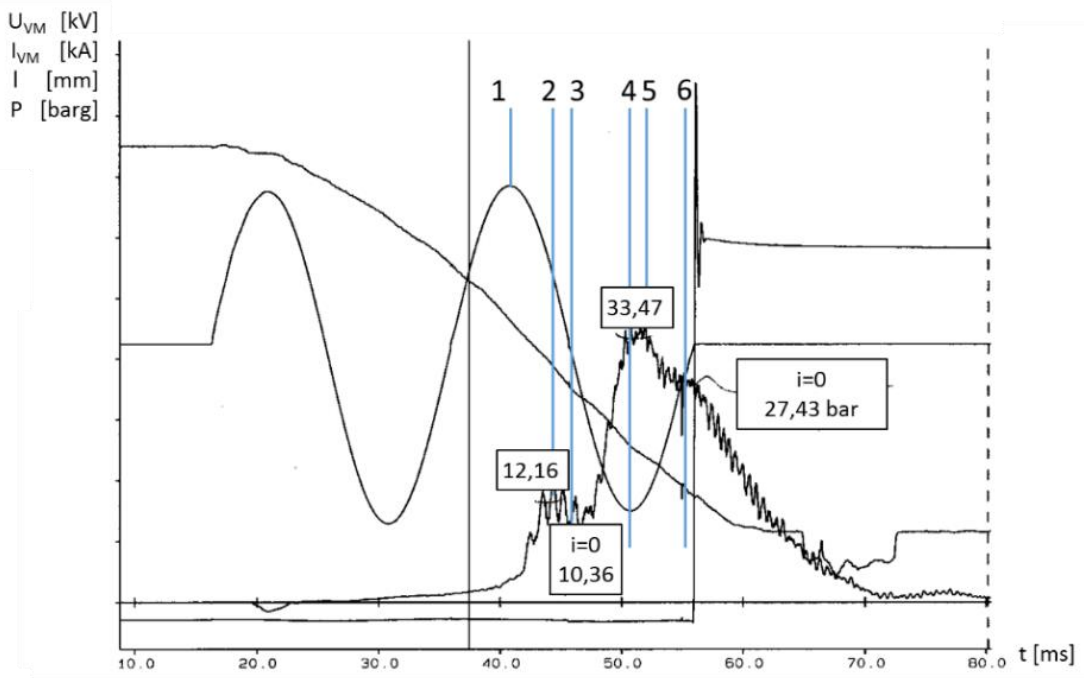

Figure 11

Breaking process with T100s minimum arcing time 
Figures 10 and 11 show the operation of the same circuit-breaker during a T100s breaking process. Figure 10 shows the maximum while Figure 11 shows the minimum arcing time. The current through the tested circuit-breaker, the transient recovery voltage, travel-time characteristic, and the pressure evolution in the thermal chamber show in the diagrams. Points 1 and 4 show the current's first and second peak values after contact separation. The pressure peak values also appear (points 2 and 5). The time shift of pressure peak values in relation to the current peak values is the consequence of the arc thermal inertia. Point 3 indicates the current's zero before breaking. Point 6 shows the start of the operation of the current injection circuit [14], [15].

The diagrams (Figure 10, Figure 11) highlight two substantial facts:

- First is that the pressure peak value arises after the current peak value. This is the effect of the arc inertia.

- Second is that the thermal chamber pressure at the maximum arcing time is lower $(8,86$ barg on Figure 10) then in case of the minimum arcing time (27,43 barg on Figure 11).

Due to the facts described in Chapter 3, the Author transforms the engineering task into a mathematical task with the use of the feedback method discussed in Chapter 4. Accordingly, the Author studied the $M_{n, 1} . . M_{n, 16}$ values where for any $E_{1}$ $\mathrm{R}_{1}=1$. On the other hand, the $\mathrm{M}_{\mathrm{n}, 1} . . \mathrm{M}_{\mathrm{n}, 16}$ values were also examined for the case where $\mathrm{p}$ provides an ideal distribution.

\section{Conclusions}

With the assistance of measurement theory and considering the standards' specifications, in this article the Author determined those typical mechanical parameters of the arcing chamber of circuit-breaker which have the greatest influence on the circuit-breaker's operation. These are the bases on which $\mathrm{A}_{\mathrm{TM}}$ matrix consisting of the mechanical parameters is set. The modification of $A_{T M}$ matrix resulted in the test parameter matrix including $E_{1} E_{9}$. The Author denominated 11 result parameters that used during the feedback process. From all of this data, the $\mathrm{A}_{\mathrm{TR}}$ result matrix is set and with the connection and aggregation of all matrixes a final matrix is set. This is the aggregated circuit-breaker development matrix. In the article, the interpretation of one test results is shown based on the oscillogram of two arcing time of T100s test.

In the following article the changes in the geometry will be associated with the pressure wave peak values. The Author will weigh the $A_{T R}$ mechanical parameters to be fed back by using Fuzzy logic. This is necessary as the FEM calculation will be fine-tuned with a feedback appropriate to CBDa model and these results will be used in the establishment of the new geometry. The Author will present the multidimensional matrix established from the aggregated matrixes used for the different tests. 


\section{Acknowledgement}

The research was supported by AD\&TE research group of Faculty of Electrical Engineering of Óbuda University.

\section{References}

[1] Dr. Madarász György Attila: "Breackers. Part 1: High voltage circuitbreaker" Elektrotechnika 2016/1-2, pp. 15-19

[2] International Electrotechnical https://webstore.iec.ch/publication/62278, 2019.03.02, 10:24

Commission,

[3] István Vajda, Alexander Glazyrin, Irina Ustinova, Evgeniy Bolovin: "Influence of Design Methods a Discrete Model of Separately Excited DC Motor on Parameters Estimation" Acta Polytechnica Hungarica Vol. 15, No. 6, 2018, pp. 219-233

[4] Zsolt, Mitrik; Péter, Kádár "System theory approach of SF6 high voltage circuit breaker development process" SAMI 2018 : IEEE $16^{\text {th }}$ World Symposium on Applied Machine Intelligence and Informatics: Dedicated to the Memory of Pioneer of Robotics Antal (Tony) K. Bejczy: proceedings Seattle (WA), United States of America : IEEE (2018) pp. 137-142, 6 p.

[5] László Horváth, Imre J. Rudas: "Information Content Driven Model for Virtual Engineering Space" Acta Polytechnica Hungarica, Vol. 15, No. 2, 2018, pp. 7-32

[6] Jana Moravčíková, Peter Pokorný: "The Influence of Machine-Part Measuring Strategies for Coordinate Measuring Devices on the Precision of the Measured Values" Acta Polytechnica Hungarica, Vol. 15, No. 6, 2018, pp. 7-26

[7] Dr. Kopper Bence, Methodology of scientific research, tf.hu/wpcontent/uploads/.../Tudományos-kutatásmódszertan.pptx, Viewing date: 2019.04.19, 16:51

[8] Halász-Huba: Technical measurments. Technical University Publishing Office 2003. ISBN 96342074]

[9] Kourosh Mousavi Takami, Erik Dahlquist "Modeling and simulation of short circuit current and trv to Develop a synthetic test system for circuit breakers" The $55^{\text {th }}$ Conference on Simulation and Modelling (SIMS 55) 2122 October, 2014, Aalborg, Denmark, Page: 285 -

[10] Park, Byung-Rak, Korea Electrotechnology Research Institute: “Type test certificate" 2011.04.22. Doc. No.: 2011TC00238

[11] Z. Conka, M. Kolcun, G. Morva, „Utilizing of Phase Shift Transformer for increasing of Total Transfer Capacity" In: Acta Polytechnica Hungarica, Vol. 13, Issue:5, pp. 27-37, 2016, DOI: 10.12700/APH.13.5.2016.5.2, ISSN: $1785-8860$ 
[12] Zsolt Mitrik, "Operation of third generation sulphur- hexafluoride circuit breakers in medium zone of short circuit currents", $32^{\text {nd }}$ Kandó Conference 17 of November 2016, ISBN 978-963-7158-07-0

[13] R. Andoga, L. Madarász, L. Főző, T. Lazar, V. Gaspar, "Innovative Approaches in Modeling, Control and Diagnostics of Small Turbojet Engines" In: Acta Polytechnica Hungarica, Vol. 10, Issue:5, pp. 27-37, 2013, DOI: 10.12700/APH.10.05.2013.5.6, ISSN: 1785-8860

[14] Rümpler Ch,-Narayanan V.R.T.: Arc Modelling Challenges, Plasma Physics and Technology, 2015, Vol. 2, No. 3, pp. 261-270

[15] Niu Ch. at al.: Simulation and Experimental Analysis of Arc Motion Characteristics in Arc Circuit Breaker, Plasma Physics and Technology, 2016, Vol. 18, No. 3, p 